\title{
Marco de referencia para la incorporación de Cobots en líneas de manufactura
}

\section{Framework for Cobots' incorporation in manufacturing lines}

INFORMACIÓN DEL

\section{ARTÍCULO}

Fecha de recepción: 1 de Septiembre de 2020.

Fecha de aceptación: 1 de Diciembre de 2020
1 Doctor in Business Administration, Atlantic International University. Docente- investigador, Universidad del Salvador- Argentina.

E-mail: sergio.salimbeni@usal.edu.ar Código ORCID:

https://orcid.org/0000-0002-7365-1053

$\overline{2}$ Estudiante de Licenciatura en Ciencias Económicas y Empresariales, Universidad del Salvador-Argentina.

E-mail: daniel.mamani@usal.edu.ar Código ORCID:

https://orcid.org/0000-0001-9219-3054

Sergio Salimbeni ${ }^{1}$, Daniel Mamani ${ }^{2}$

\section{Resumen}

El propósito de este estudio es el desarrollo de un marco de referencia para la evaluación y análisis de viabilidad de la incorporación de robots colaborativos en líneas de manufactura. Este trabajo se ha realizado en dos fases: (1) se hizo una revisión de la literatura en lo referente a la Transformación Digital y la Industria 4.0 y sus tecnologías asociadas y (2) se estudiaron las técnicas existentes para el análisis de incorporación de robots colaborativos en estaciones de trabajo, desarrollándose luego un marco de referencia para el análisis de factibilidad. Se concluye que una de las maneras más convenientes es evaluarlo desde 5 factores: el aspecto técnico, ergonométrico, de calidad, el punto de vista económico financiero y regulatorio, siendo este último un aporte del presente trabajo de investigación.

\section{Palabras Clave:}

Cuarta Revolución Industrial, Industria 4.0, Transformación Digital, Pymes, Robots, Producción, Toma de Decisiones multicriterio.

Clasificación JEL: O3, O39.

\begin{abstract}
The aim of this study is the development of a framework for the evaluation and feasibility analysis for the incorporation of collaborative robots in the manufacturing lines. This work has been carried out in two phases: (1) a review of the literature regarding Digital Transformation and Industry 4.0 and its associated technologies was carried out; (2) existing techniques were studied for analysis of collaborative robots' incorporation in workstations; a reference framework was later developed for feasibility analysis. It is concluded that a 5 -factor analysis is one of the most convenient evaluation ways: technical, ergonometric and quality aspects, economic-financial and regulatory viewpoint. The latter point is a contribution to current research work.
\end{abstract}

CITACIÓN: Salimbeni, S., \& Mamani, D. (2020). Marco de referencia para la incorporación de Cobots en líneas de manufactura. Podium, 38, 159-180. doi:10.31095/podium.2020.38.10

ENLACE DOI:

http://dx.doi.org/10.31095/podium.202 0.38 .10

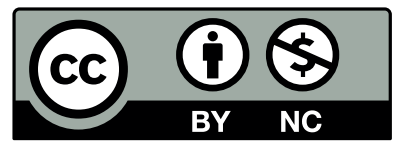

\section{Keywords:}

Fourth Industrial Revolution, Industry 4.0, Digital Transformation, SMEs, Robots, Production, Multicriteria Decision Taking.

JEL Classification: O3, O39. 


\section{Introducción}

El comportamiento del consumidor está cambiando y existen nuevas tendencias que deben ser analizadas. Este patrón de comportamiento puede representar nuevas áreas de oportunidades en los negocios. El consumidor moderno tiende a tomar en cuenta diversos factores: el ecológico, la conectividad, la responsabilidad social o la salud y el tipo de producto. Las empresas se ven obligadas a agregar valor, el cual debe ser percibido por los clientes, obteniendo de ese modo una ventaja competitiva (Xin y Ojanen, 2018). En los países desarrollados, alrededor del $25 \%$ de los hogares son unipersonales. En la Argentina es alrededor del $20 \%$ mientras que en la Ciudad de Buenos Aires es del 36,8\% (INDEC, 2017). Existen nuevas maneras de relacionarse, conectarse o informarse. Los ciclos de vida de los productos son más cortos y los clientes consumen productos más complejos y personalizados.

Basado en dichos cambios, el sector de la producción también está transitando por un nuevo paradigma. Se agrega a ello la situación actual generada por la pandemia que está obligando al distanciamiento social, menor cantidad de trabajadores en las plantas y en las empresas en general, $y$, consecuentemente, el trabajo a distancia. Todo ello requiere, de parte de los fabricantes, pasar de una producción masiva a una producción personalizada, efecto que se está evidenciando ya desde hace algunos años. Esto último, a su vez, obliga a las fábricas a rediseñar sus líneas de producción en término de: (1) lotes, (2) variedad de productos y (3) tiempos de puesta en el mercado (Krajewski, Ritzman, y Malhotra, 2008). La trasformación digital aplicada a la industria, denominada Industria 4.0 (I4.0), mejora esos tres factores. La colaboración entre los humanos y los robots es una de las características salientes de la I4.0 y es por ello por lo que se necesita una herramienta que permita evaluar la conveniencia, o no, de la incorporación de robots colaborativos (Cobots) en las líneas o estaciones de manufactura. Se presenta en este estudio un marco de referencia para dicha evaluación.

\section{Revisión de literatura}

Transformación Digital e Industria 4.0

\section{Inicios}

La transformación digital es el cambio asociado con la aplicación de tecnologías digitales en todos los aspectos de la sociedad humana. El término Digital proviene de dígito, y se refiere al sistema binario, y la acción de la conversión a dicho sistema se lo denomina Digitalización.

En 1703, Gottfried Wilhelm von Leibniz, matemático alemán, desarrolló el concepto que sería conocido como "digitalización", el cual publicó en su artículo "Explication de l'Arithmétique Binaire". Estaba desarrollado como un sistema numérico en base-2, o sea, que representaba dos valores: 1 ó 0 . El sistema luego fue mejorado $\mathrm{y}$ complementado por George Boole 
(1854), matemático británico (álgebra de Boole), Claude Elwood Shannon (1938), matemático e ingeniero estadounidense reconocido por su obra un libro titulado "La teoría matemática de la comunicación" y George Stibitz, científico estadounidense conocido por sus trabajos realizados en los años 1930 y 1940 por el desarrollo de circuitos digitales lógicos, usando relés electromecánicos como conmutadores (Baum y Warwick, 1997).

\section{Primeras computadoras}

La primera computadora electrónica, analógica, fue presentada por John Atanasoff, de origen búlgaro, en 1939. Con una subvención y la asistencia de un estudiante graduado, Clifford Berry, construyó el prototipo de la computadora Atanasoff Berry Computer (ABC) en noviembre de ese año. Stibitz es también considerado uno de los pioneros de la computación digital, a través del desarrollo de la primera computadora electromecánica, la cual funcionaba con relés. El proceso de la digitalización se aceleró con el desarrollo de computadoras personales como el Simon en 1950, Apple II en 1977 e IBM PC en 1981. Con la introducción de la World Wide Web (www), cambiaron fundamentalmente el alcance, la dimensión, la escala, la velocidad y los efectos de la digitalización, dando lugar a una mayor aceleración al proceso de transformación de las sociedades (Ford y Baum, 1997).

\section{Digitización y Digitalización}

La "Digitización" se puede definir como la conversión de información analógica a digital, es decir, a formato binario. Ésta implica la representación de señales, sonidos, imágenes y objetos con valores discretos. Prácticamente todos los sectores industriales y de servicios han sido fuertemente favorecidos por esta conversión de los datos. A diferencia de la Digitization, la Digitalización se define como el "proceso" del cambio inducido dentro de las industrias en general. Este proceso ha facilitado el desarrollo de varios de los fenómenos conocidos actualmente como el IoT (Internet de Cosas), Internet de las Cosas Industrial (IIoT), Big data (BD), Blockchain e Inteligencia Artificial (AI). Un error que se encuentra habitualmente es definir a la digitalización como el uso intensivo de Tecnologías de la Información (TI); sin embargo, es un cambio aún más profundo, que transforma no sólo a la empresa, sino que trae cambios organizacionales y sociales (Ford y Baum, 1997).

\section{Transformación digital e Industria 4.0}

La Transformación Digital (TD) se describe como "el efecto social global de la digitalización". La digitización sustenta el proceso de digitalización, que dio lugar a mayores oportunidades para transformar y cambiar los modelos de negocio, las estructuras socioeconómicas, las medidas legales y políticas, los patrones organizacionales y las barreras culturales existentes hoy en día. Por lo tanto, la digitización (la conversión), la digitalización (el proceso) y la TD (el efecto), aceleran los procesos de cambio en la sociedad. Más allá de la implementación tecnológica o la 
digitalización de procesos y/o servicios, la TD implica un cambio de mentalidad y la creación de una cultura de transformación que empatice con dicho cambio y esté dispuesta a aceptarlo (Colombo, et al., 2014).

La incorporación de Cobots en la industria está enmarcado en la 4ta Revolución Industrial, o sea, la I4.0, que es un concepto relativamente nuevo que también recibe otras denominaciones, tales como Industria digital, Fabricación inteligente, confundiéndose también con la 4ta revolución industrial o TD. En verdad, aunque desde una perspectiva pragmática los conceptos 4ta Revolución Industrial e I4.0 tiendan a significar lo mismo, desde lo académico y en estricto rigor conceptual, se encuentran algunas diferencias.

Hacia fines del siglo XVIII, con la invención de la máquina a vapor, se dice que comienza la ler revolución industrial. En el siglo XIX, con el uso industrial de la electricidad se inicia la 2da. La denominada 3ra revolución industrial, a mediados del siglo $\mathrm{XX}$, fue determinada por la invención del semiconductor y las técnicas digitales (Salimbeni y Bianchi, 2019).

La 4ta Revolución Industrial se refiere a un fenómeno tecnológico, social, económico, político y cultural, que se ha venido gestando desde la 1ra originada en Europa en el siglo XVIII y que dio origen a una sociedad urbanizada e industrializada. Es en este contexto y con la aparición de un conjunto de nuevas tecnologías aplicadas a la Industria, iniciadas con Internet y luego la AI (Acosta, 2019), el IIoT, la Computación en la nube (CC) y los sistemas ciberfísicos (CPS), diversos autores definieron una nueva evolución del desarrollo industrial el cual denominaron: "Cuarta Revolución Industrial" (Rauch, 2020; Matt, Modrák, y Zsifkovits, 2020; Qin, Liu, y Grosvenor, 2016; Sanders, Elangeswaran, y Wulfsberg, 2016).

Por su parte, la I4.0 se refiere a la implementación práctica y sistemática de los principios de la "Cuarta Revolución Industrial" en las industrias de transformación, a través de procesos de producción y gestión, buscando mayores niveles de productividad, automatización, virtualización, instantaneidad y modularización. Se podría resumir diciendo que la I4.0 es la TD aplicada a la industria. La I4.0 implica la completa digitalización de las cadenas de valor, a través de la integración de tecnologías de procesamiento de datos, software inteligente y sensores, de modo de poder monitorear, predecir, controlar, planear y producir, de forma inteligente, lo que genere mayor valor a toda la cadena. El IIoT, la AI y los CPS son los pilares de la I4.0 ( Ishiguro, Yamaoka, Kanda, Mutlu, y Hagita, 2009).

\section{La Fábrica Inteligente}

Las tecnologías digitales se han estado convirtiendo en factores clave para aquellas empresas que buscan alcanzar sus metas (Nylén y Holmström, 2015). En la feria de Hannover 2011, por primera vez, se mencionó a este fenómeno como "Industry 4.0" (Rauch, 
2020; Matt et al., 2020; Qin et al., 2016; Sanders et al., 2016).

El término I4.0 describe la revolución de la industria manufacturera alrededor del mundo. El foco de la I4.0 es la combinación de la producción, las tecnologías de la información e Internet (Matt et al., 2020). Es la integración e interacción de tecnologías, tanto en los dominios digitales como físicos, lo cual la diferencian de otras revoluciones industriales (Demartini \& Tonelli, 2018); los objetos inteligentes se comunican entre sí y se crea una Internet de objetos y servicios. El mundo físico y el mundo virtual se integran en sistemas ciberfísicos (Sommer, 2015).

En los últimos años se han diferenciado dos niveles de la I4.0 (Rauch, 2020). El primer nivel de la I4.0 es principalmente impulsado por la tecnología y es la base para el segundo nivel, denominado I4.0+ el cual incorpora la AI.

Las tecnologías asociadas a la I4.0 se pueden resumir en las siguientes (Lydon, 2019):

1. Computación en la nube: uso de software, aplicaciones, servicios y datos compartidos entre diferentes ubicaciones y sistemas alojados de forma remota.

2. Tecnologías móviles: es una tecnología que va donde va el usuario. Consiste en dispositivos portátiles de comunicaciones bidireccionales, dispositivos informáticos y la tecnología de red que los conecta.

3. Comunicación Máquina-Máquina: es un concepto genérico que se refiere al intercambio de información o comunicación en formato de datos entre dos máquinas remotas.

4. Manufactura aditiva: la impresión aditiva o 3D, es un grupo de tecnologías de fabricación por adición donde un objeto tridimensional es creado mediante la superposición de capas sucesivas de material.

5. Robótica avanzada: son robots capaces de trabajar sin una supervisión humana y a su vez automatizar otros robots para que sean también automáticos.

6. Big Data y analítica: se refiere a un conjunto de datos un volumen y complejidad de tal magnitud que precisan de aplicaciones informáticas no tradicionales.

7. Internet de las Cosas Industrial (IIoT): se refiere a la interconexión entre objetos físicos en un ámbito industrial, a través de dispositivos electrónicos que permiten la colecta y el intercambio de datos.

8. Computación Cognitiva: se refiere al uso de hardware y / o software que imita el funcionamiento del cerebro humano ayudándolos a la toma de decisiones. (Lee, Davari, Singh, y Pandhare, 2018).

9. Ciberseguridad: es la práctica de proteger computadoras, servidores, dispositivos móviles, sistemas electrónicos, redes y datos de ataques maliciosos. También se la conoce como seguridad de la tecnología de la información o seguridad de la información electrónica.

10. Sistemas ciberfísicos CBS: un sistema ciberfísico es un mecanismo controlado o monitorizado por algoritmos 
basados en computación y estrechamente integrados con Internet, donde colaboran los sistemas mecánicos con los informáticos (Colombo et al., 2014).

\section{Sistemas de Fabricación}

El sistema de fabricación es una integración entre equipos y recursos laborales, que puede llevar a cabo una o más actividades de producción a partir del material, partes o conjunto de partes. El sistema de fabricación está influenciado por varios factores, los cuales son tipos de operaciones, número de estaciones de trabajo, nivel de automatización y flexibilidad del sistema. En base a estos factores se definen seis tipos generales de un sistema de fabricación: (1) celdas manuales de estación única, (2) celdas automatizadas de estación única, (3) sistemas de ensamble manual, (4) sistemas de ensamble automatizado, (5) sistemas de fabricación por celdas y (6) sistemas de fabricación flexibles. Además de estos seis sistemas de fabricación típicos, existen muchos otros definidos por los especialistas, tales como: el sistema de fabricación integrado por computadora y el sistema de fabricación reconfigurable. Con estos sistemas de fabricación principales se ha concebido la Industria 4.0. (Qin et al., 2016).

Una fábrica inteligente es aquella capaz de conectar y compartir información de forma omnidireccional entre todas sus personas y objetos (cosas), con la finalidad de obtener un sistema que logre alinear la demanda, el diseño, la fabricación y el suministro. La conectividad e interoperabilidad, tanto vertical como horizontal, son la calve de la fábrica inteligente (Leitão, Colombo, y Karnouskos, 2016). Ella debe alcanzar todas las operaciones y áreas productivas, tanto de la empresa como de los proveedores y clientes, es decir, a lo largo de toda la cadena de valor, donde personas y máquinas puedan interactuar ágilmente.

La selección del tipo de distribución depende, en gran parte, de la estructura de los procesos, es decir, de la posición de los procesos en la matriz de contacto con los clientes y proveedores de servicio y en la matriz de productos y procesos para los procesos de manufactura. Existen cuatro tipos básicos de distribución: (1) flujo flexible; (2) flujo en línea; (3) híbrida, y (4) posición fija. Los procesos de trabajo con flujos muy divergentes tienen bajo volumen y un alto nivel de personalización. Para tales procesos, el gestor debe elegir una distribución de flujo flexible, en la que los recursos humanos y materiales se organizan por función en lugar de por servicio o producto. La distribución de flujo flexible es habitual cuando en la misma operación se deben fabricar varios productos $o$ partes distintos, o es preciso atender a muchos clientes diferentes de forma intermitente (Krajewski et al., 2008).

Las ventajas de la distribución de flujo flexible sobre la distribución de flujo en línea, donde los centros están dispuestos en una trayectoria lineal, son las siguientes: los recursos son de propósito general y menos intensivos en capital; existe mayor flexibilidad para manejar 
los cambios en la mezcla de productos; la supervisión de los empleados es más especializada cuando el contenido del trabajo requiere una buena dosis de conocimientos técnicos y la utilización del equipo es mayor. Cuando los volúmenes son bajos, dedicar recursos en forma exclusiva a cada producto $o$ servicio, como se hace en la distribución de flujo en línea, requeriría más equipos para agrupar todos los requisitos para todos los productos. Un desafío importante cuando se diseña una distribución de flujo flexible, consiste en localizar los centros de tal manera que impongan cierto orden.

\section{Robots colaborativos}

La palabra "Robot" fue introducida en la literatura en 1920, en la obra R.U.R. (Rossum's Universal Robots), de Čapek (2019), nacido en lo que hoy es la República Checa. Proviene de la palabra checa "robota", que significa "labor forzada, servicio, esclavo". Este nombre fue utilizado en el imperio austrohúngaro hasta 1848. La palabra inventada por Josef Čapek sirve para designar a las máquinas trabajadoras o serviles. E1 "Cobot", por su parte, es un brazo robótico creado para trabajar junto a los humanos en una cadena de producción, de ahí que sean conocidos como robots colaborativos o "Collaborative Robots".

Los Cobots y los robots industriales tradicionales se diferencian en varios aspectos. El robot industrial produce de forma masiva, ocupa mucho espacio y, en general, permanece en una posición fija. En cambio, el Cobot es compacto, ocupa poco espacio y se puede reubicar en cualquier lugar de la planta con relativa facilidad. Los robots industriales, por su actividad intensiva, resultan peligrosos. Debido a ello no pueden instalarse sin barreras de seguridad que protejan a las personas. La ausencia de sensores de fuerza, que dota al robot de la capacidad de conocer su entorno y que le permite detenerse automáticamente en caso de una intrusión en su espacio, sumado a su elevado peso, convierte a los robots industriales en herramientas potencialmente peligrosas. Por el contrario, los Cobots, están equipados con sensores que les permiten detenerse en caso de obstrucción o necesidad. En conclusión, los Cobots ofrecen la oportunidad de eliminar las barreras de seguridad para llevar a las personas al núcleo del proceso productivo y facilitar su integración en una cadena de producción. La mayor diferencia entre los Cobots y los robots industriales es el objetivo con que cada uno de ellos fue diseñado. Un robot industrial se imagina, planifica, construye y utiliza con un único propósito y ese propósito lo podrá resolver de manera eficiente. El Cobot puede estar desarrollado para realizar una sola tarea o para realizar varias según las necesidades de producción. Se puede reprogramar de forma sencilla para que realice una nueva serie de tareas de forma rápida y sin la necesidad de ayuda de un experto en robótica. Por último, mientras que el robot hace su trabajo en forma autónoma, el Cobot trabaja como colaborador del operario y no como sustituto (Rauch et.al, 2018).

Salimbeni y Bianchi (2019) señalan 
que son varias las ventajas de trabajar junto a un Cobot; por ejemplo, son capaces de hacerlo las 24 horas ininterrumpidamente, están preparados para ejercer la fuerza requerida para las tareas más pesadas y realizan labores repetitivas en forma eficiente, dotando al producto de la mejor calidad, permitiendo a las empresas alcanzar elevados niveles de productividad. La combinación de la tecnología de producción convencional con las TICs, la M2M con el IIoT y los Cobots, transforman las plantas en fábricas inteligentes; todo esto gracias a la colaboración del robot con el hombre (ver Figura1).

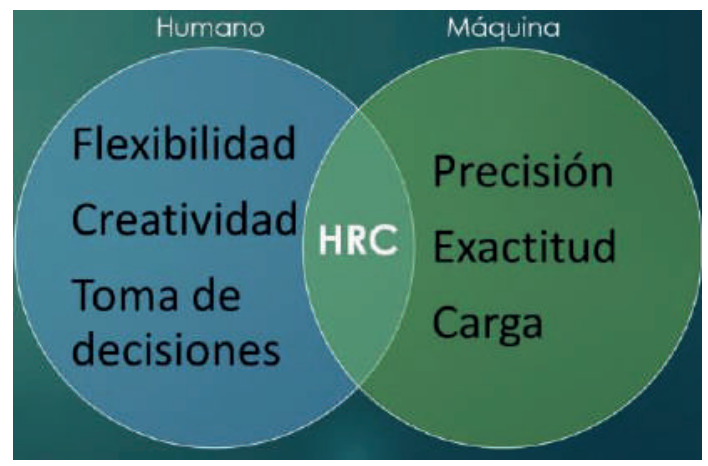

Figura 1. Interacción Humano-Máquina.

Fuente: Elaboración propia.

Conforme Rauch (2020), uno de los especialistas mundiales en el tema, las ventajas de la colaboración entre humanos $\mathrm{y}$ robots (HRC) son las siguientes:

- Mejoran las condiciones de trabajo de los operarios debido a los cuidados en seguridad.

- Mejoran la utilización de las áreas de producción debido a que no existen barreras físicas.

- Mejoran la accesibilidad a las áreas de trabajo.
- Aumentan la capacidad de producción y calidad.

- Mejoran la utilización de la fuerza laboral más especializada.

La HRC permite también una división de tareas "dinámica" y una revisión del cronograma de trabajo en tiempo real (Rauch, 2020). Otro de los beneficios clave es la posibilidad de transformar estaciones de trabajo actuales en sistemas colaborativos. Esto ofrece escalabilidad y se precisaría menor inversión por el hecho de ser una mejora y no un rediseño total de la planta.

La pregunta habitual es si es posible y razonable implementar una estación de trabajo colaborativo a partir de los puestos de ensamble que las fábricas usan en la actualidad. Ello es posible y debe evaluarse cada caso desde diferentes perspectivas; inclusive, esta interacción trae aparejados también cambios en la estructura organizacional de la empresa, los cuales deben ser analizados.

La colaboración humano-robot en tareas de ensamble, requiere que ambas partes puedan hacer referencia a objetos en un espacio compartido. Los humanos pueden usar una combinación de descripciones verbales, gestos señaladores y miradas para seleccionar uno de los muchos objetos. Es posible que un humano, que esté en una tarea de ensamble, no pueda hacer gestos para indicar a un Cobot la siguiente pieza. Sin embargo, dado que las descripciones verbales pueden ser difíciles de interpretar, el Cobot necesita formas de lograr una base común de qué objeto 
pretendía el usuario. Las expresiones de referencia ambiguas se pueden resolver mediante una base verbal, señalando o utilizando la mirada. Por supuesto, los Cobots también pueden usar formas no humanas para indicar su foco de atención, como proyectar sus necesidades en el entorno compartido o mediante el uso de realidad aumentada o mixta. Se debe tomar en consideración las ventajas del robot y del trabajador (Kragic, Gustafson, Karaoguz, Jensfelt, y Krug, 2018), tal como se muestra en la Tabla 1.

Tabla 1.

Características del Humano y Robot

\begin{tabular}{cc}
\hline HUMANO & ROBOT \\
\hline Intuición & Velocidad \\
Flexibilidad & Manejo de grandes \\
Percepción & volúmenes y pesos \\
Pensamiento & Alcance \\
Decisión & Reproductibilidad \\
Actuación & Precisión \\
\hline
\end{tabular}

Fuente: Elaboración propia.

Se ha podio evidenciar, en varios experimentos que, debido a las características del humano y los robots, el primero se enfoque mucho más en actividades de monitoreo y control, mientras que las tareas rutinarias y de precisión, queden en mano de los robots colaborativos. Esto es válido para cualquier tipo de línea de manufactura (Kragic et al., 2018).

\section{Metodología}

La metodología utilizada se basa en un enfoque cualitativo y desde la perspectiva de la ingeniería industrial, es decir, desde una visión más técnica que económica. El alcance de la investigación es exploratorio y descriptivo. $\mathrm{La}$ metodología, para el marco teórico ha sido búsqueda y gestión bibliográfica con fichas de lectura, método de mapeo y uso del método pentágono.

Se realizó en dos fases (ver Figura 2): (1) se realizó una revisión de la literatura en lo referente a la Industria 4.0 y sus tecnologías y (2) se profundizó el estudio de líneas de la automatización en pymes y robots colaborativos, generando un marco de referencia y conjunto de herramientas para la evaluación de su posible incorporación en estaciones manufactureras.

En la primera fase se relevaron 328 documentos de los cuales fueron seleccionados 48 para su análisis, estudiando 29 de ellos en particular; uno de los más importantes fue el German Standarization Roadmap Industrie 4.0 versión 3. Dicho trabajo se realizó del siguiente modo: (1) En bases de datos académicas (Researhgate, Scielo, Google Académico y Science Direct) con cadena de búsqueda a través de la combinación del operador "o" entre las palabras clave (4th Industrial Revolution, Industry 4.0, Industrie 4.0, Digital Transformation, SMEs, Robots, Production, Multicriteria Decision Making), se recogieron las referencias que cumplían con los siguientes criterios: (a) fueron publicados en actas de congresos, artículos, revistas, series de libros y libros entre los años 2017 y 2020; (b) contenían, al menos, uno de los términos de búsqueda en el resumen, título y / o palabras clave. (2) $\mathrm{Se}$ eliminaron los duplicados. (3) $\mathrm{Se}$ descartaron los que no tenían a disposición textos completos. (4) $\mathrm{Se}$ 
excluyeron los documentos que definieron Industria 4.0 fuera del alcance de este trabajo de investigación. (5) Se clasificaron conforme las preguntas de investigación. (6) $\mathrm{Se}$ analizaron los documentos colectados y recopilaron los datos correspondientes de interés para las preguntas de investigación.

En la segunda fase se generó, fundamentalmente a partir de experiencias europeas, una guía de referencia para la evaluación de integración de Cobots en las Pymes.

\section{Resultados}

Estudio de viabilidad para la incorporación de un Cobot en una estación de trabajo

La decisión de la incorporación de Cobots en estaciones de trabajo no sólo deben tomarse en base a un criterio económico, sino que son decisiones multicriterio (Benotsmane, Kovács, y Dudás, 2019; Munzer, Toussaint, y Lopes, 2017; Gombolay, Bair, Huang, y Shah, 2017). De acuerdo a lo estudiado, quien mejor lo expresa y con un método sistemático son Gualtieri, Rauch, Rojas,
Vidoni, y Matt (2018), quien sugiere evaluar cuatro factores como base para la toma de dicha decisión. Ellos son:

1. Evaluación Técnica.

2. Evaluación antropocéntrica.

3. Evaluación de la calidad del proceso y el producto.

4. Evaluación económica.

Dadas las peculiaridades de nuestra región (Latinoamérica), se ha decidido incorporar un quinto factor, el cual es de vital importancia para las empresas, el cual hemos denominado: Regulaciones.

\section{Factor I: Evaluación Técnica}

El análisis de la viabilidad técnica para la introducción de un Cobot tiene como objetivo investigar si una tarea puede ser realizada por él de manera eficiente, considerando sus limitaciones técnicas de hardware y / o software. En general, es necesario verificar si cierto tipo de Cobot, equipado con dispositivos estándar, puede realizar la alimentación, manipulación y/o montaje de los componentes involucrados en una línea de producción mediante el uso de recursos adecuados.
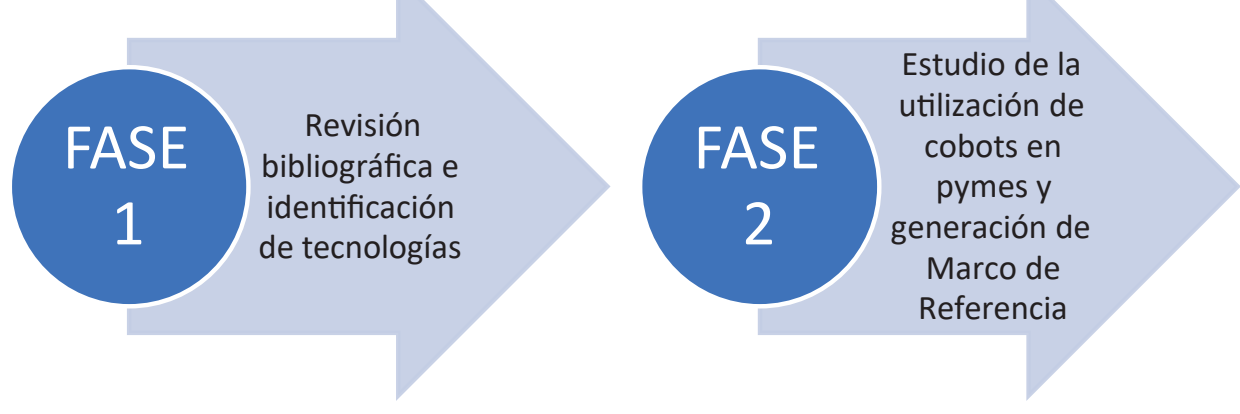

Figura 2. Metodología.

Fuente: Elaboración propia. 
Para la evaluación técnica se sugiere el siguiente proceso de análisis que se presenta en la Figura 3 (Rauch, Matt, Brown, Towner, Vickery, y Santiteerakul, 2018):

- Estudiar si el componente y / o la secuencia de ensamble requieren dos manos para su manejo.

- Analizar si el componente y / o la secuencia de ensamble requiere habilidades humanas típicas (por ejemplo, percepción táctil, audición y capacidad de interpretar situaciones (Gualtieri et al., 2018).

Durante este diagnóstico técnico, existen cuestiones críticas respecto de: (1) la alimentación, (2) el manejo y (3) el ensamble. Algunas de las más importantes son:

\section{Alimentación:}

- El componente es magnético o pegajoso.

- El componente es un nido o enredo.

Manejo:

- El componente no tiene eje de simetría.

- El componente es frágil o delicado.
- El componente es flexible.

- El componente es muy pequeño o grande (en referencia a una mano humana).

- El componente es liviano, por lo que la resistencia del aire crearía problemas en el transporte.

- El componente es resbaladizo.

Ensamble:

- Los componentes no tienen una "superficie de referencia" que simplifique el posicionamiento preciso durante el montaje.

- Los componentes no pueden orientarse fácilmente.

- Los componentes no incluyen características que permitan la auto-alineación durante el montaje.

\section{Factor II: Evaluación ergonométrica}

\section{Principios Ergonométricos}

En la I4.0, el papel de los humanos dentro de los sistemas de trabajo sociotécnicos es especialmente digno de atención. Ya sea como actor en el proceso

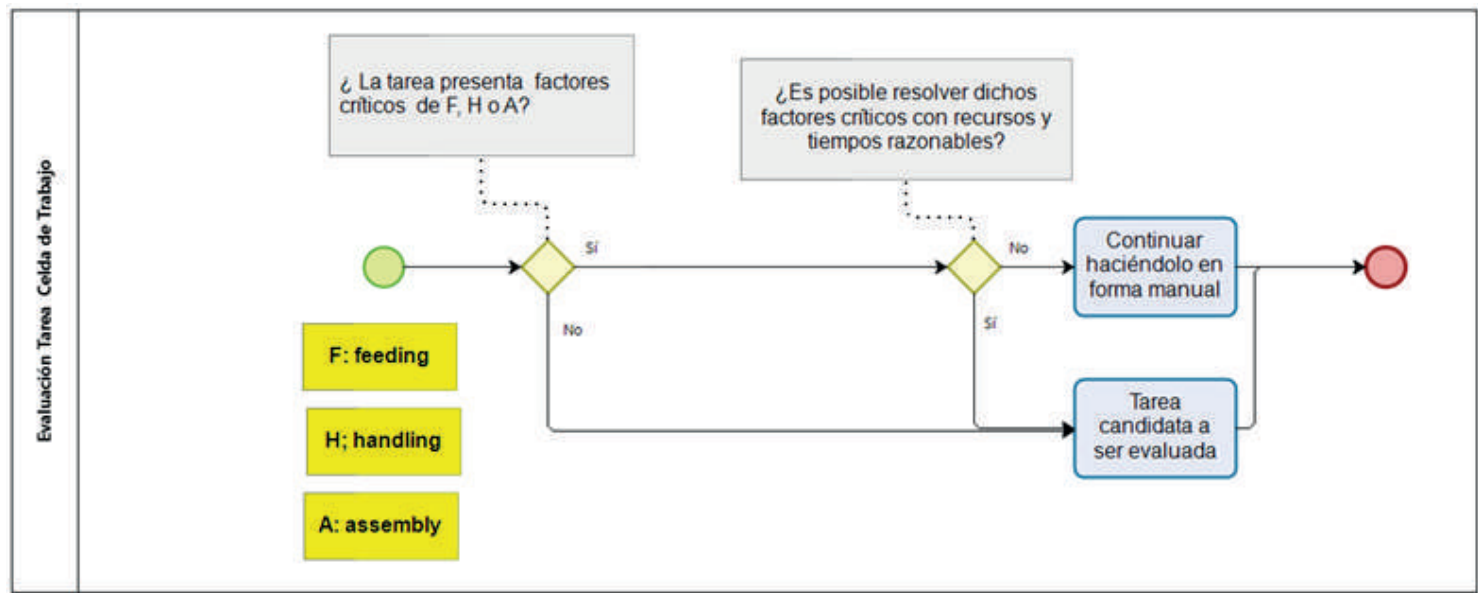

Figura 3. Flujograma de evaluación de tareas.

Fuente: (Rauch et al., 2018). 
de producción, operador de máquinas u operador de mantenimiento, planificador de producción o programador, el hombre continuará ocupando roles clave dentro del proceso de producción. Para diseñar un sistema de trabajo que sea ergonómico, eficiente y flexible, pero que también sea exitoso a largo plazo, es importante que las personas se incluyan en el proceso de diseño. Con ese fin, se pueden emplear los criterios ampliamente aceptados para el trabajo amigable con los humanos. Estos deben regir todas las acciones del proceso de estandarización relacionadas con la ergonomía (DKE, 2020).

La Ergonomía es una disciplina que busca que los humanos y la tecnología trabajen en completa armonía, diseñando y manteniendo los productos, puestos de trabajo, tareas y equipos de acuerdo con las características, necesidades y limitaciones humanas. La norma DIN ISO 6385 se ocupa de los principios ergonómicos para el diseño de sistemas de trabajo (Secretaría de Salud Laboral de CCOO de Madrid, 2016).

El sistema de trabajo es entendido como una combinación de personas y equipos de trabajo que actúan juntos en un proceso laboral, para una finalidad expresa, en un lugar de trabajo y en un ambiente bajo condiciones impuestas por las tareas a realizar. Por esto, se consideran: (1) Tarea Laboral, (2) Equipamiento de Trabajo, (3) Procesos de Trabajo, (4) Ambiente de Trabajo, (5) Angustia laboral, (6) Tensión por el trabajo y (7) Fatiga.
En esta fase, el análisis y evaluación ergonométrica tiene como objetivo cuantificar los beneficios relativos que el operador obtendrá desde el punto de vista del esfuerzo físico a partir del trabajo colaborativo con un robot.

Existen diferentes análisis o métodos de evaluación ergonométricos que se pueden realizar a modo de diagnóstico, con el objetivo de determinar cuáles son aquellos puestos o células de trabaja aptos para la incorporación de un robot colaborativo. Algunos pueden ser (Femeval, 2016):

- JSI (Job Strain Index o Índice de Tensión o Esfuerzo): creado por Moore J.S. y Gard A. Se basa en la observación directa (video) con el objetivo de valorar aquellos puestos de trabajo donde el trabajador está más expuesto a padecer desordenes traumáticos acumulativos, en las partes distales de los miembros superiores (mano, muñeca, antebrazo, codo) debido a movimientos repetitivos.

- ERGO IBV: desarrollado por Instituto Biomecánico de Valencia. Esta herramienta es que permite evaluar, de manera individual, los riesgos ergonómicos en una amplia variedad de tareas, como: manipulación manual de cargas simple y múltiple, manipulación manual de cargas para trabajadores lesionados, tareas repetitivas, posturas forzadas, psicosocial, diseño antropométrico del puesto de trabajo.

- NIOSH (National Institute for Occupational Safety and Health): esta ecuación permite evaluar aquellas tareas 
donde se realizan esfuerzos para levantar peso, brindando el peso máximo con el objetivo de prevenir patologías lumbares y dolores de espalda.

- Snook \& Ciriello - "The design of manual handling tasks": este método incluye un conjunto de tablas con los pesos máximos aceptables, tanto para hombre como mujer, para diferentes acciones como levantamiento, el descenso, empuje, arrastre y transporte de carga.

- OCRA (Occupational Repetitive Actions): busca evaluar el riesgo por manipulación repetitiva a alta frecuencia, en relación con la maquinaria utilizada y las tareas que provocan lesiones en los miembros superiores, teniendo en cuenta frecuencia de movimiento, posturas y movimientos forzados. Hay una versión de este método más simplificada conocida como Check-list OCRA.

- RULA: Rapid Upper Limb Assessment: se base en evaluar una o varias posturas concretas, que puedan llegar a generar trastornos en los miembros superiores, ya sea por su duración o por la carga postural que esta conlleva. Es un método que se basa en la observación durante varios ciclos de trabajo.

\section{Análisis y prevención de riesgos}

Las herramientas de una Industria 4.0 están divididas en dos grandes grupos: las tecnologías que basan su funcionamiento en captar y procesar datos y las herramientas físicas, tangibles $\mathrm{y}$ perceptibles. Se enfoca en el segundo grupo, con el objetivo de determinar y prevenir los posibles riesgos que la incorporación de una estas herramientas puedan provocar.

Existen riesgos que se pueden presentar de manera general en cualquiera de las dos tecnologías mencionadas anteriormente, independientemente de la que se esté aplicando (Secretaría de Salud Laboral de CCOO de Madrid, 2016). Estos pueden ser:

- Riesgo en cuanto a la planificación y organización necesaria para que la empresa puede incorporar una nueva tecnología.

- Riesgos psicosociales, o sea, aquellas dificultades que puedan alterar la integridad del personal, a nivel psicológico, emocional y social.

- Riesgos de seguridad, higiene y ergonomía, que son aquellos que impactan negativamente sobre la integridad física de los empleados, derivados de la interacción directa o indirecta con la nueva tecnología.

- Ciberseguridad: al existir una conectividad entre todas las máquinas y un intercambio constante de datos, existen ciertos factores que pueden aumentar drásticamente la vulnerabilidad de la base de datos de la empresa.

\section{Recomendaciones de la DIN/DKE roadmap}

Al diseñar espacios de trabajo y estaciones de trabajo, se debe considerar 
el despliegue potencial de nuevas tecnologías como exoesqueletos y ortesis. Estos son capaces de apoyar al operador en diversas situaciones, como cuando se requiere una fuerza corporal particular. Debe verificarse si los datos existentes relacionados con la fuerza muscular deben complementarse para abordar este tipo de soporte. También debería examinarse la posibilidad de que robots móviles, colaborativos, exoesqueletos / ortesis o anteojos inteligentes puedan conducir a un conjunto completamente nuevo de riesgos. Deberán definirse requisitos para elementos tales como rutas de escape o el techo permisible en el entorno de trabajo real. También deberán definirse los requisitos para la seguridad de estas tecnologías y su interacción con el espacio de trabajo. Las tecnologías informáticas móviles a menudo se mencionan en relación con la I4.0. Estos hallazgos también deben reflejarse en el diseño (Electrical et al., 2014, p.116).

Factor III: Evaluación de la calidad del proceso y del producto

El término "Calidad" es muchas veces empleado con el significado de excelencia de un producto o servicio (Oakland, 2002, p.15). Calidad es simplemente el cumplimiento de las exigencias del cliente, lo cual ha sido expresado de diversas maneras por distintos autores.

La calidad 4.0 (Q4.0) es la adaptación y mejora de los principios de la calidad, debido a, y con el fin de, aplicarlos a las nuevas fábricas, las fábricas inteligentes.
La gestión de la calidad en la I4.0 es denominada Q4.0. No es una tecnología sino un proceso utilizado para maximizar el valor para los usuarios (Jacob, 2018). La Q4.0 es una innovación de la Gestión de la Calidad Total, tal como se observa en la Figura 4.

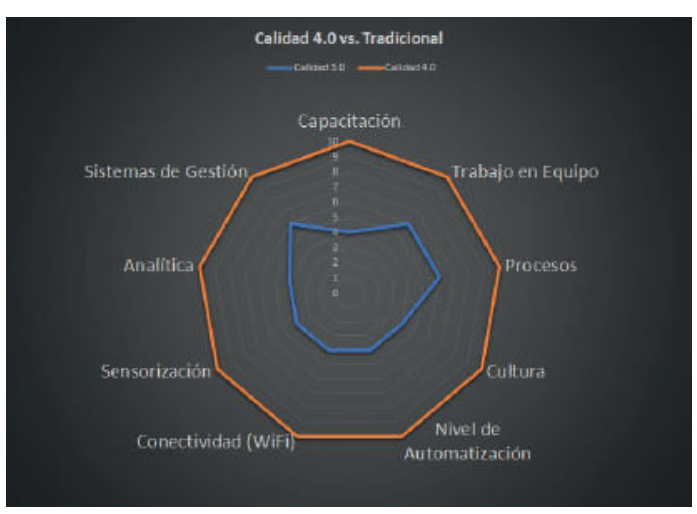

Figura 4. Calidad tradicional vs. Q4.0.

Fuente: Elaboración propia.

No sólo el proceso industrial y el producto mejoran, sino también la calidad de la información; esto es debido a los nuevos métodos de captura y análisis de datos horizontal (Illés, Tamás, Dobos, y Skapinyecz, 2017). “... un sistema de información es un conjunto de componentes interrelacionados, desarrollados para colectar, procesas, almacenar $\mathrm{y}$ distribuir información para facilitar la coordinación, el control, análisis, visualización y el proceso de decisiones" (Simoes Gomes; 2002; p.125).

La fortaleza de la conexión digital, el servicio y los dominios físicos, definirá, en última instancia la calidad de la información necesaria para la planificación, operación y optimización del proceso. El motivo es que cuando los datos se transmiten en tiempo real, la transparencia y la calidad de la 
información aumentan notablemente. Al adquirir y procesar la información del producto con alta calidad se permite un monitoreo y una conciencia automáticos que producen como resultado un aumento en la eficiencia de todos los elementos del concepto de I4.0. Con ese fin, se debe evaluar para cada tarea las posibles mejoras en la calidad del proceso y del producto, visando las reducciones de la variabilidad y la inestabilidad (Rauch et al., 2018).

La posibilidad de tener digitalizado gran parte del proceso de trabajo en las celdas mejorará notablemente la calidad de la información, ya que permitiría el monitoreo y control en tiempo real de las diferentes actividades, tal como se muestra en la Figura 5 (Ansari, Glawar, y Nemeth, 2019).

\section{Factor IV: Evaluación económica y financiera}

Este análisis consiste en la evaluación de los factores económicos y financieros de la compañía, la estabilidad y la realización de beneficios de una opción de inversión. Incluye una consideración del costo total de la mejora a realizar, así como los costos y beneficios totales del uso y soporte de la solución. Los analistas de negocios utilizan el análisis financiero para hacer una recomendación de solución para una inversión de un proyecto de cambio o mejora específica, al comparar una solución o enfoque de solución con otras, basándose en el análisis de:

- costo inicial y el período de tiempo en que se incurre en esos costos,

- beneficios financieros esperados y el marco temporal en el que se incurrirán,

- costos continuos de usar la solución y respaldar la solución, riesgos asociados con el proyecto de cambio o mejora, y

- riesgos continuos para el valor comercial de usar esa solución.

Se utiliza habitualmente una

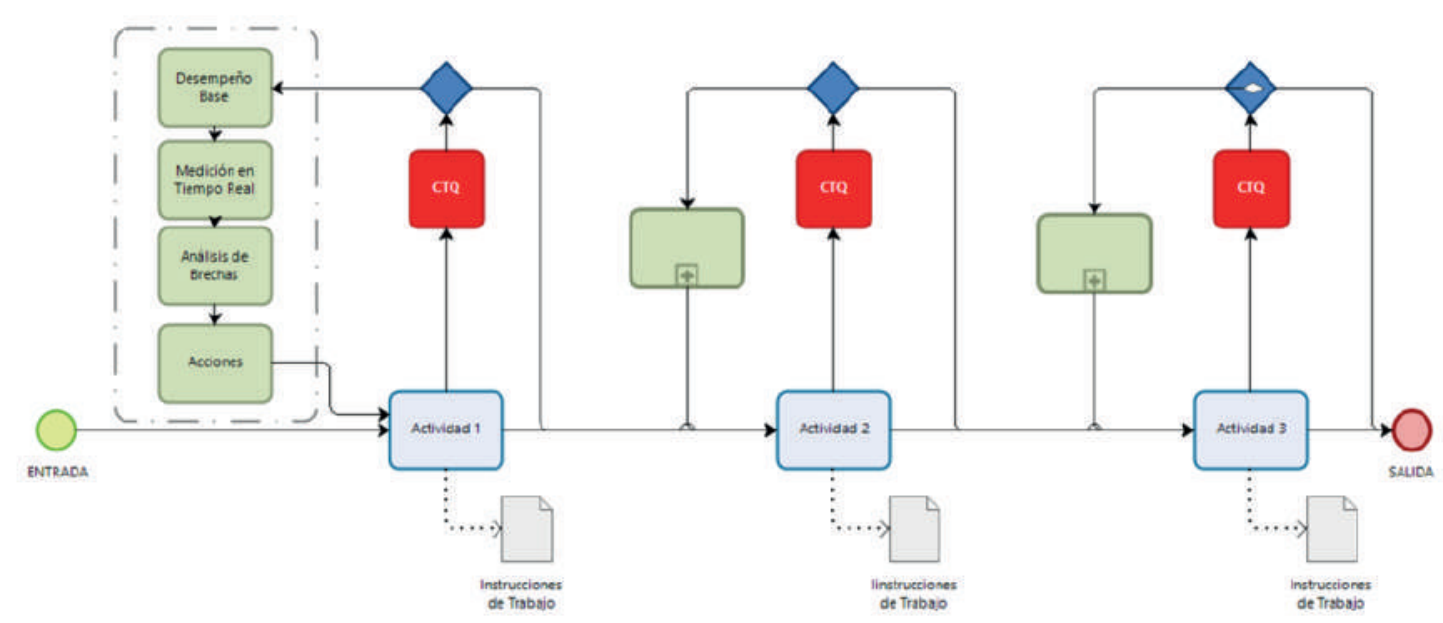

Figura 5. Control de calidad anticipativo.

Fuente: Elaboración propia. 
combinación de técnicas de análisis, ya que cada una proporciona una perspectiva diferente. Los ejecutivos comparan los resultados del análisis financiero de una opción de inversión con el de otras posibles inversiones para tomar decisiones sobre cuál alternativa sería la más recomendable. El análisis financiero aborda la incertidumbre $\mathrm{y}$, a medida que un proyecto de cambio o mejora progresa a lo largo de su ciclo de vida, los efectos de esa incertidumbre se entienden mejor.

Se deben tomar en consideración diferentes clases de costos:

\section{Costo de cambios}

El costo de un cambio incluye el costo esperado de construir o adquirir los componentes de la solución, en este caso de la inclusión de un Cobot en una celda de trabajo, y los costos esperados de la transición de la empresa desde estado actual al futuro. Esto podría incluir los costos asociados con el cambio de equipos, software, instalaciones, personal $\mathrm{y}$ otros recursos, contratos existentes, subsidios, multas, conversión de datos, capacitación, comunicación del cambio y gestión del despliegue. Estos costos pueden compartirse entre organizaciones dentro de la empresa.

\section{Costo de propiedad}

El costo total de propiedad (TCO-Total Cost of Ownership) es el costo de adquirir una solución, el costo de usar la solución y el costo de apoyar la solución en el futuro previsible, combinados para ayudar a comprender el valor potencial de dicha solución. En el caso de equipos e instalaciones, a menudo existe una esperanza de vida generalmente aceptada. Algunas organizaciones asumen un período de tiempo estándar de entre tres y cinco años.

\section{Realización del valor}

El valor generalmente se realiza con el tiempo. El valor planificado podría expresarse anualmente o podría expresarse como un valor acumulado durante un período de tiempo específico.

\section{Análisis Costo Beneficio}

El análisis de costo-beneficio es una predicción de los beneficios totales esperados respecto de los costos insumidos. Las suposiciones sobre los factores que componen los costos y los beneficios se deben indicar claramente en los cálculos para que puedan revisarse, cuestionarse y aprobarse. Los costos y beneficios a menudo se estimarán en función de esos supuestos, y la metodología de estimación debe describirse para que pueda ser revisado y ajustado si fuera necesario.

El período de tiempo de un análisis de costo-beneficio debe mirar hacia el futuro lo suficiente como para que la solución esté en pleno uso y el valor planificado se esté logrando. Esto ayudará a comprender cuáles costos se incurrirán y cuándo debe realizarse el valor esperado.

\section{Cálculos Financieros}

Las organizaciones usan una combinación 
de cálculos financieros estándar para comprender diferentes perspectivas sobre cuándo y cómo las diferentes inversiones entregan valor. Estos cálculos tienen en cuenta los riesgos inherentes a las diferentes inversiones, la cantidad de dinero inicial que se invertirá en comparación con el momento en que se realizarán los beneficios, una comparación con otras inversiones que la organización podría hacer y la cantidad de tiempo que tomará recuperar Inversión original. Entre ellos se encuentran: el período de recupero (PB) el valor actualizado neto (VAN) el índice de retorno (IR) y la tasa interna de retorno TIR.

\section{Factor V: Leyes y Regulaciones}

Se incorpora, en el marco de referencia desarrollado en el presente trabajo, la perspectiva de la seguridad e higiene y sus normas y regulaciones asociadas.

Se detallan posibles riesgos a presentarse en diferentes áreas, provocados por la incorporación de Cobots u otras nuevas tecnologías físicas y tangibles, existen riesgos específicos al momento de incorporarlos en las estaciones de trabajo.

Los posibles riesgos que acarrea la incorporación de Cobots, se analizan tomando en consideración las siguientes áreas: a) Seguridad, b) Higiene c) Ergonomía y d) Psicosociales (Femeval, 2016).

\section{Seguridad}

Contactos mecánicos que generen daños físicos por acceso a zonas peligrosas durante el funcionamiento automático. Contactos mecánicos que generen daños físicos durante ajustes, programación o pruebas del robot. Contactos mecánicos que generen daños físicos durante la limpieza o mantenimiento, provocado por arranques o puesta en funcionamiento inesperados. Contactos mecánicos que generen impactos o daños físicos, debido a que el movimiento del robot excede su área de trabajo. Contacto mecánico que genere impactos o daños físicos durante el trabajo colaborativo. Proyecciones de Materiales (Partículas, chispas, salpicaduras). Riesgos Eléctricos (Contacto con partes activas que generen quemaduras, electrocución) y durante el mantenimiento (elementos mal aislados, voltaje incorrecto, soldaduras de alto voltaje). Riesgos Térmicos (quemaduras, atmosfera inflamable o explosiva, polvo metálico).

\section{Higiene}

Exposición a vibraciones perjudiciales para la salud del empleado o que pongan en peligro funcionamiento el robot. Exposición a radiaciones (ionizantes y no ionizantes). Presencia de productos o materiales propios del proceso perjudiciales a la salud (pintura, elementos químicos, polvo). Exposición prolongada al propio ruido del proceso o del robot. Exposición a campos electromagnéticos.

\section{Ergonomía}

Aparición de movimientos repetitivos en área mano-brazo, provocado por manipulación de piezas, herramientas y otros. Aparición de sobreesfuerzos en la manipulación manual de cargas pesadas, 
durante la carga y descarga de piezas y elementos pesados, entre otros. Mantener por tiempo prolongado posturas forzadas al momento de realizar mantenimiento, fases de espera, accionar mandos $\mathrm{y}$ controles. Aparición de cambios posturales debido a reducción la libertad de movimiento, limitaciones en el espacio de trabajo, no poder adoptar posturas y posiciones habituales.

\section{Psicosociales}

Rechazo hacia la tecnología robótica. Generación de un sentimiento de dependencia, provocando en el empleado una pérdida de identidad y autonomía. Cambios en el ritmo y organización del trabajo. Sobrecarga y fatiga mental (Femeval, n.d.).

\section{Análisis de viabilidad}

Para dar respuesta a la pregunta inicial, de si es viable o no la incorporación de un Cobot en una celda o línea de producción, se procede a la toma de decisión en base a la selección u orden de preferencia de las diferentes alternativas, para lo cual se consideran, cinco criterios centrales: (1) técnico, (2) ergonométrico, (3) de calidad, (4) económico y (5) regulatorio. Los primeros cuatro factores son los utilizados por los especialistas antes mencionados, y este 5to factor es el aporte de este trabajo considerando la importancia del mismo en Latinoamérica.

Esto es lo que se conoce como una toma de decisiones multicriterio, ya que se debe seleccionar o priorizar una de entre varias alternativas, tomando en consideración más de una variable o criterio.

Para estos casos de toma de decisiones multicriterio, se propone el uso de los métodos Direct rating, AHP o Promethee. El direct rating es el más simple, el AHP (de la escuela americana) es bien práctico y ordenado cuando se cuenta con gran cantidad de subcriterios de selección y finalmente el Enfoque en Preferencia /Indiferencia (Outranking) (Escuela francesa), ya sea el PROMETHEE, Preference Ranking Organization Method for Enriched Evaluation o el ELECTRE, Elimination Et Choix Traduisant la Realité / Elimination and Choice, los cuales tienen como ventaja que evitan el efecto de compensación en la puntuación de criterios, permitiendo su comportamiento

Tabla 2.

Matriz para la toma de decisión de incorporación de un cobot

\begin{tabular}{llll}
\cline { 2 - 3 } & \multicolumn{1}{c}{ Estación Manual } & Cobot tipo 1 & Cobot tipo 2 \\
\cline { 2 - 3 } Factores & & \\
\hline Factor I: Técnico & \\
\hline Factor II: Ergonométrico & \\
\hline Factor III: Calidad & \\
\hline Factor IV: Económico & \\
\hline Factor V: Regulatorios & \\
\hline Fuente: Elaboración propia.
\end{tabular}


pueda ser diferente y por lo tanto incomparable, y dado que las preferencias no pueden ser sumadas o compensadas matemáticamente, es posible seleccionar un mayor grado de preferencia a una opción cuando no son comparables en la realidad.

Se ejemplifica considerando tres alternativas, de entre las cuales se debe seleccionar la mejor de ellas, evaluando los 5 criterios antes mencionados. Para ello se construye la matriz de decisión, tal como se observa en la Tabla 2.

\section{Conclusiones}

El comportamiento del consumidor está cambiando, lo cual obliga a los fabricantes a pasar de una producción masiva a una personalizada. Debido a ello las configuraciones de planta deben modificarse, requiriendo mayor flexibilidad y mejorando su desempeño. Los robots colaborativos, gracias a su rápida reconfiguración y adaptabilidad, entre otras virtudes, juegan un papel clave en esta trasformación.

La decisión de incorporar un cobot en una celda de trabajo no se basa solo en el grado de madurez de la empresa ni exclusivamente en la necesidad de mejorar el desempeño. Conforme los marcos de referencia estudiados, la decisión de incorporación de cobots debe considerar diversos criterios. Los especialistas coinciden en evaluar cuatro aspectos: los técnicos, ergonométricos, de calidad y económicos. Las características socioeconómicas y de idiosincrasia de la Argentina y países de la región, deben considerar un quinto factor: el de las regulaciones laborales, higiene y seguridad. Se proponen en este trabajo dos mejoras a dichos marcos de referencia: la incorporación de dicha quinta variable y un método sistemático de evaluación para esta toma de decisión multicriterio. Se propone para un próximo estudio, el análisis de un caso práctico utilizando este marco de referencia adaptado.

\section{Referencias}

Acosta, M. (2019). Inteligencia artificial: la cibernética del ser vivo y de la máquina. Naturaleza y Libertad. Revista de Estudios Interdisciplinares, (12), 13-30. https://do i.org/10.24310/natylib.2019.v0i12.6262

Ansari, F., Glawar, R., y Nemeth, T. (2019). PriMa: a prescriptive maintenance model for cyber-physical production systems. International Journal of Computer Integrated Manufacturing, 32(4-5), 482-503. https://doi.org/10.1080/095119 2X.2019.1571236

Baum, M. S., y Warwick, F. (1997). Secure electronic commerce. New Jersey: Prentice Hall.

Benotsmane, R., Kovács, G., y Dudás, L. (2019). Economic, social impacts and operation of smart factories in Industry 4.0 focusing on simulation and artificial intelligence of collaborating robots. Social Sciences, 8(5), 1-20. https://doi.org/10.3390/socsci 8050143

Capek, K. (2019). R. U. R. :Rossum's Universal Robots. Digireads.com Publishing.

Colombo, A.W., Bangemann, T., Karnouskos, S., Delsing, J., Stluka, P., Harrison, R., Jammes, F., y Lastra, J. L. (2014). Industrial Cloud-Based Cyber-Physical Systems: The IMC-AESOP Approach. Springer. 
Demartini, M., y Tonelli, F. (2018). Quality management in the industry 4.0 era. Proceedings of the XXIII Summer School Francesco Turco- Industrial System Engineering.

DKE - Deutsche Kommission Elektrotechnik Elektronik Informationstechnik. (2020). German Standardization Roadmap Industrie 4.0. Recuperado de https://www.din.de/blob/65354/5721876 7bd6da1927b181b9f2a0d5b39/roadmap-i 4-0-e-data.pdf

Femeval (n.d.). R-Evolución Industrial: Más Segura Más Productiva Más Humana Prevención $Y$ Retos 4.0. Recuperado de https://www.femeval.es/dam/jcr:59be7c2 8-1c13-4d42-b77c-62120f061075/GUIA $\% 20$ PREVENCION\%20Y\%20RETOS\% 204.0.pdf

Femeval. (2016). Más Humana Robots Industriales $Y$ Cobots. https://www.femeval.es/dam /jcr:fd091e5f-3c97-42fd-9981-680e8232 a645/Guia_Robots.pdf

Ford, W., y Baum, M. S. (1997). Secure Electronic Commerce (1ra ed.). New Jersey: Prentice-Hall, Inc.

Gombolay, M., Bair, A., Huang, C., y Shah, J. (2017) Computational design of mixed-initiative human-robot teaming that considers human factors: situational awareness, workload, and workflow preferences. The International Journal of Robotics Research, 36(5-7), 597-617. https:// doi.org/10.1177/0278364916688255

Gualtieri, L., Rauch, E., Rojas, R., Vidoni, R., y Matt, D. T. (2018). Application of Axiomatic Design for the Design of a Safe Collaborative Human-Robot Assembly Workplace. MATEC Web of Conferences, 223. https://doi.org/10. 1051/matecconf/201822301003

Illés, B., Tamás, P., Dobos, P., y Skapinyecz, R. (2017). New challenges for quality assurance of manufacturing processes in industry 4.0. Solid State Phenomena, 261, 481-486. https://doi.org/10.4028/www. scientific.net/SSP.261.481

Instituto Nacional de Estadística y Censos de la República Argentina. (2010). Característica de la población y sus hogares. Ciudad de Buenos Aires. Recuperado de https://www.indec.gob.ar/

Ishiguro, H., Yamaoka, F., Kanda, T., Mutlu, B., y Hagita, N. (2009). The Industrial Internet of Things Volume G1: Reference Architecture. Industrial Internet Consortium, 1.80, (69).

Jacob, D. (2018). Quality 4.0 Series: The Quality Leader's New Product Introduction Playbook.

Kragic, D., Gustafson, J., Karaoguz, H., Jensfelt, P., y Krug, R. (2018). Interactive, collaborative robots: Challenges and opportunities. IJCAI International Joint Conference on Artificial Intelligence Proceedings, 18-25. https://doi.org/10.24963/ijcai.2018/3

Krajewski, L., Ritzman, R., y Malhotra, M. (2008). Administración de Operaciones: Procesos y Cadenas de Valor. México: Pearson Educación.

Lee, J., Davari, H., Singh, J., y Pandhare, V. (2018). Industrial Artificial Intelligence for industry 4.0-based manufacturing systems. Manufacturing Letters, 18 , 20-23. https://doi.org/10.1016/j.mfglet. 2018.09.002

Leitão, P., Colombo, A. W., y Karnouskos, S. (2016). Industrial automation based on cyber-physical systems technologies: Prototype implementations and challenges. Computers in Industry, 81. https:// doi.org/10.1016/j.compind.2015.08.004

Lydon, B. (2019). RAMI 4.0 reference architectural model for Industrie 4.0. InTech, 66(2). 
Matt, D.T., Modrák, V., y Zsifkovits, H. (2020). Industry 4.0 for SMEs. Challenge, Opportunities and Requirements. Springer Link. https://doi.org/10.1007/ 978-3-030-25425-4

Munzer, T., Toussaint, M., y Lopes, M. (2017). Preference learning on the execution of collaborative human-robot tasks. Proceedings-IEEE International Conference on Robotics and Automation, 879-885. https://doi.org/10.1109/ICRA. 2017.7989108

Nylén, D., y Holmström, J. (2015). Digital innovation strategy: A framework for diagnosing and improving digital product and service innovation. Business Horizons, 58(1), 57-67. https://doi.org/ 10.1016/j.bushor.2014.09.001

Oakland, J.S. (2002). Gerenciamento da qualidade total. São Paulo, Brasil: Nobel.

Qin, J., Liu, Y., y Grosvenor, R. (2016). A Categorical Framework of Manufacturing for Industry 4.0 and Beyond. Procedia CIRP, 52, 173-178. https://doi.org/10.1016/ j.procir.2016.08.005

Rauch, E., Matt, D. T., Brown, C. A., Towner, W., Vickery, A., y Santiteerakul, S. (2018). Transfer of industry 4.0 to small and medium sized enterprises. Advances in Transdisciplinary Engineering, 7, 63-71. https://doi.org/10.3233/978-1-61499-898 $-3-63$

Rauch, E. (2020) Industry 4.0+: The Next Level of Intelligent and Self-optimizing Factories. In: Ivanov V., Trojanowska J., Pavlenko I., Zajac J., Peraković D. (eds) Advances in Design, Simulation and Manufacturing III. DSMIE 2020. Lecture Notes in Mechanical Engineering. Springer, Cham. https://doi.org/10.1007/978-3-030 -50794-7_18

Salimbeni, S., y Bianchi, S. (2019). Estado actual y evolución de la industria nacional hacia la industria 4.0. Innova UNTREF Revista Argentina en Ciencia y Tecnología, 3. https://www.untref.edu.ar/innova/en_curs o_2.php

Sanders, A., Elangeswaran, C., y Wulfsberg, J. (2016). Industry 4.0 implies lean manufacturing: Research activities in industry 4.0 function as enablers for lean manufacturing. Journal of Industrial Engineering and Management, 9(3), 811-833. https://doi.org/10.3926/jiem.1940

Secretaría de Salud Laboral de CCOO de Madrid. (2016). Métodos de evaluación ergonómica. Comisiones Obreras de Madrid Salud Laboral de Madrid. Recuperado de http://www.madrid.ccoo. es/54c00d40d3dea466094a35e6b6a867d 9000045.pdf

Simões Gomes, F.C. (2002). Gestão da cadeia de suprimentos integrada à tecnologia da informação. São Paulo, Brasil: Thomson

Sommer, L. (2015). Industrial revolution-Industry 4.0: Are German manufacturing SMEs the first victims of this revolution? Journal of Industrial Engineering and Management, 8(5), 1512-1532. https://doi.org/10.3926/jiem.1470

Xin, Y., y Ojanen, V. (2018). The impact of digitalization on product lifecycle management: How to deal with it? IEEE International Conference on Industrial Engineering and Engineering Management, 2017-Decem, 1098-1102. https://doi.org/10.1109/IEEM.2017.8290 062 
